\title{
Precision Medicine in the Context of Artificial Intelligence
}

\begin{tabular}{|r|l|}
\hline Journal: & SIGMOD Record \\
\hline Manuscript ID & SR-2019-0004 \\
\hline Manuscript Type: & Research Articles \\
\hline Date Submitted by the \\
Author: & 25-Oct-2019 \\
\hline Complete List of Authors: & $\begin{array}{l}\text { Rayan, Rehab; Alexandria University High Institute of Public Health, } \\
\text { Epidemiology }\end{array}$ \\
\hline
\end{tabular}

\section{SCHOLARONE \\ Manuscripts}




\title{
Precision Medicine in the Context of Artificial Intelligence
}

\author{
Rehab Rayan \\ High Institute of Public Health/ Alexandria University \\ Alexandria, Egypt \\ rayanr@alexu.edu.eg
}

\begin{abstract}
Similar to all fields of study, growth occurs with breakthrough or developmental research spanning to the progress and intimate utility of technology. Dilemmas of various sectors have been favorably resolved by adopting artificial intelligence (AI) algorithms. Applying Precision Medicine profoundly depends on AI algorithms to work Precision Medicine queries like; predicting or detecting, diagnosing the disease properly, and optimizing therapy, hence, the selection of the algorithm is affected by its capacity and practicality. Nevertheless, it is yet in its initial step and fronts some hurdles crucial to the flourishing deployment of precision medicine like research, adoption values, and authority controls. Notwithstanding, Precision Medicine also pretends some difficulties like; modifying the health discipline and profession to the fact that automata and algorithms could displace most of the healthcare professional tasks they act now. Ultimately, effective employment of precision medicine would rescue countless lives and improve the health profession. This review examines the present state of AI applications in precision medicine and future opportunities. It discusses major AI systems like IBM Watson, examining moral accountability and legal obligations when applying it in clinical decision-making, advantages and boundaries of employing Watson and different AI clinical decision support techniques, and considerations before consulting AI systems.
\end{abstract}

\section{INTRODUCTION}

A medication might treat an individual while not work for another, or resulting in adverse events in others. Furthermore, some people get conditions such as neoplasms, unlike others who do not. Genomic composition and some other agents like living habits or age might cause such issues. Medicine better tackle every patient's condition distinctly by customized therapies to the individual's biology and history; that is called Precision or Personalized Medicine [1].
Patients with comparable diagnoses should not be addressed alike; they may get other medications for effective care. Precision medicine is an arm of medicinal disciplines that applies clinical recommendations and experience to provide personalized medical service to patients. Precision medicine can anticipate the potential of a person for an illness, attain a reliable diagnosis, and provide the top therapeutic options [2]. Hereditary data is a core component in individualizing clinical care. Nonetheless, the bulk of iterative remarks indicate that rare genomic diversity, not mutual genes, is responsible for the inherent preservation from or predisposition to a medical condition.

Applying Artificial Intelligence in renovating precision medicine is crucial regarding personalization and specificity of finding illness and adopting care plans. It is important to manage enzymes' metabolism and medication side effects that develops in some individuals with problems in removing medicines out of their bodies, thus resulting in intoxication; meantime others get rid of the medicine prior functioning [3]. Today, clinics' and hospitals' automation via computers for recording clinical tasks or adopting electronic medical record (EMR) systems; offers clinical data and information which might be utilized as a basis to improve healthcare delivery.

\section{PRECISION MEDICINE INTELLIGENCE}

Public health intelligence is a cornerstone and a key leader for personalized healthcare approach [4]. Precision medicine is an advance for managing health conditions, which takes into account hereditary, lifestyle, environmental and personal variance. For instance, the All of Us Research Program, a project by the National Institute of Health (NIH), shoots for initiating a novel framework of patient-centered study for evolving medical alternatives to reach the optimal conclusions for forecasting epidemics, avoiding or curing medical conditions, and boosting living quality 
[5]. In order to address various matters with precision medicine, information technology should expand to strengthen liaison, involvement, and cooperation among patients, families, medical societies, and clinical squad entailing professionals from diverse disciplines. For instance, mutual decision-making is endorsed for rising patient interaction, linking care to the patient's expectations, adhering regimens and better health results [6].

Artificial intelligence is lately employed to work on individualized data; extract patient choices; facilitate patients and families taking part in the healthcare process and allow clinicians delivering competent and superior quality customized healthcare by tailoring broad treatment strategies and attaching patients with data ahead of those found inside the clinical scene [7]. Community health specialists and caregivers may utilize the technology to provide personalized measures and treatments founded on the finest prove to establish outstanding patient care and wholesome people. For instance, machine learning (ML) can refine prognosis reliability for Stage II colorectal malignancy [8].

\section{AI: THE ESSENCE OF PRECISION MEDICINE}

The National Institutes of Health defined precision medicine as an emerging path in preventing and treating diseases, which counts for genes, lifestyle and environmental diversity among individuals [4]. Hence, it enables investigators and physicians to anticipate with an increased precision preventing or treating plans for managing a distinct condition in specific groups of individuals. It demands supercomputers with a considerable computing potential; deep learning algorithms which learn independently at a matchless speed; and mostly AI to utilize health professionals cognitive skills at a novel level.

Supercomputers computing potentials' have grown into an arena for countries to show their strength off [9]. Deep learning algorithms have been taught to give judgments at least as oncologists, cardiologists and dermatologists [10-12]. Nonetheless, it is worth mentioning the significance of coupling health practitioners' knowledge with the before-mentioned algorithms. During the great challenge of the International Symposium on Biomedical Imaging, opponents designed computational systems to identify metastatic breast carcinoma in full slide pictures of biopsies from the sentinel lymph node. The leading algorithm possessed a $92.5 \%$ success rate; meanwhile, a pathologist examined the corresponding pictures with a $96.6 \%$ success rate. Consequently, consolidating the judgment from a human pathologist with the predictions from the deep learning system improved the pathologist's success rate to $99.5 \%$ with nearly $85 \%$ decline in human error rate [12].

AI is classified in general into three levels: artificial general intelligence, artificial narrow intelligence (ANI), and artificial superintelligence [13]. In the near future, ANI is the most anticipated to rise. ANI can examine sets of data, extract inferences, uncover unique associations, and maintain health caregivers' work. Some companies have proved that deep learning, supercomputers, and ANI can boost precision medicine (Table 1).

Table 1. Prime companies utilizing artificial intelligence in Medicine [14].

\begin{tabular}{|c|c|c|}
\hline $\begin{array}{c}\text { Companies } \\
\text { mine medical } \\
\text { records }\end{array}$ & $\begin{array}{c}\text { Companies work } \\
\text { in biological and } \\
\text { medication } \\
\text { development }\end{array}$ & $\begin{array}{c}\text { Companies } \\
\text { work in } \\
\text { medical } \\
\text { imaging }\end{array}$ \\
\hline $\begin{array}{c}\text { Google } \\
\text { Deepmind } \\
\text { Health }\end{array}$ & Atomwise & $\begin{array}{c}\text { Butterfly } \\
\text { Network }\end{array}$ \\
\hline $\begin{array}{c}\text { IBM } \\
\text { WatsonPaths }\end{array}$ & $\begin{array}{c}\text { Recursion } \\
\text { Pharmaceuticals }\end{array}$ & 3Scan \\
\hline Careskore & Whole Biome & Enlitic \\
\hline Zephyr Health & iCarbonX & Arterys \\
\hline $\begin{array}{c}\text { Oncora } \\
\text { Medical }\end{array}$ & Deep Genomics & Bay Labs \\
\hline Sentrian & Turbine & - \\
\hline CloudMedX \\
Health
\end{tabular}

\section{AI INTEGRATION IN THE CLINICAL WORKFLOW: THE BLACK- BOX DILEMMA}

An AI application's greater attributed positive impact, than the human one, in detecting cancer cells might help to adopt it to refine pathologists' diagnosis. In this instance, employing the AI systems might assist in educating pathologists, because it maintains an increased success rate than the naked human eye. If the application detects any cancer cells that could have been simply skipped, and accordingly a patient may die, then it should be certainly used. Surprisingly, a clinic may be accused of knowing about this application while not making use of it. Unfortunately, doubts regarding the forecasting algorithms need more ethical evaluation [15].

The neural network algorithm, the backbone of AI application, detects cancer cells with an unknown mechanism and hence the black-box dilemma. Consequently, the AI application could not ascertain malignancy of specific cells. Therefore, the 
application, which is supposed to complement, instead of substituting the pathologist's role, cannot assist a pathologist to know more about malignant cells. As a result, a pathologist's role can involve discovering the shared visual characteristics between benign and malignant cancer cells and the articulating triggers in malignant cells that make them discovered by the AI application. Additionally, this role might clear up the black-box dilemma, and attain transparency that would increase patients and pathologists' confidence while depending on the AI systems. Surprisingly to clinicians, but not to patients, an AI system could be universally adopted and might boost its success rate from $92 \%$ to almost $100 \%$ with an unknown mechanism [16]. Since the task of this program is virtually black or white, detecting or denying cancer, all that matters is their success in finding cancer cells. If this is the case, with humans lagging behind, the application might substitute pathologists, letting them do other tasks where their experience is required. Eventually, the AI application operates more rapidly and is not likely to give a false diagnosis. However, the black-box dilemma might yet be crucial when there is a chance of an AI application affecting lives in a questionable manner. Thinking of self-driving cars, making lives at stake, a black-box here is related to comprehending the decisions of a self-driving car and whether they are feasible morally. Hence, the principles for decisions rendered by artificially intelligent platforms to be well-interpreted prior feeling confident utilizing them.

There is no medical or moral problem with computerization if the application attains near $100 \%$ success rate. Yet, if the success rate is less than that, it is crucial to be confident that the application retains the caliber input, which perhaps implies that the AI system learned from a set of patients with different races and ages. With guaranteed variable inputs, the AI system would hold a greater success rate in spotting cancer cells than pathologists towards precise diagnosis. Since both the pathologists and the AI application do not possess the $100 \%$ precision, it is feasible that the greatest precision might be attained when AI complements the pathologists' knowledge and skill. Probably the application would give other errors than human pathologists; therefore, utilizing both might lead to the virtually precise diagnosis [16]. From a moral position, there is some worry where the AI system has not passed across a thorough verification procedure. For instance, the success rate for an AI application has been related to that of only a single pathologist, with only a single collection of slides; hence, this reference does not provide credible evidence that the AI system's success rate would be superior to that of pathologists in a certain healthcare facility. For the AI programs' dilemmas, it might be too promising for those who are only clinically skilled to recognize what is needed to ascertain that these techniques shall be adopted. Eventually, it is advisable that both ethical, AI and clinical experiences shall be accounted for in reviewing panel recommendations concerning healthcare practice.

Designing ML patterns demand well-formatted and educated data around a gradual trend, which stays fairly balanced. A far from this overt adjusting by AI paying excessive significance to false relationships across data. For example, Google attempted to forecast the seasonal flu prevalence by just adopting the entered keywords into its search engine in 2008. For The Individuals searching behaviors vary significantly over time, the pattern was indeed an inefficient indicator for the future. Therefore, it was rapidly terminated [17]. Meanwhile, digital and anonymous data are desired for helping in research and development.

\section{CONSULTING WATSON}

IBM designed Watson, a sophisticated software responding to queries, which could be adopted as a clinical decision support system (CDSS), to help clinicians determining diagnosis and treatment alternatives. The software utilizes various AI techniques involving machine learning, natural language processing, semantic analysis, information retrieval, and automated reasoning. In 2011, the program configuration, named DeepQA (deep question and answering), notably defeated two Jeopardy game showcase competitors in a broadcast competition [18].

Watson is an augmented intelligent system, in which technology complements regular human intelligence for more precision and speed in undertaking activities [18]. Watson basically operates by feeding large quantities of unstructured and semi-structured data like examination findings (radiology results), medical records and scientific studies into its database. A clinician asks the application by outlining certain patient symptoms and other relevant elements. At the beginning, Watson examines the feed to discover the key information, subsequently; it mines a patient's data to retrieve related findings regarding the patient's genetic and medical history. Eventually, the application analyzes these data to formulate and test hypotheses and then offers a ranking of personalized, confidence-graded suggestions, like a patient's suitability for certain medications. The application utilizes many intelligent algorithms and ranking techniques to decide the confidence level in the returned proof and boost the suggested recommendations that is presented in text format [19]. Since Watson is continually being fed with data, the application could learn gradually to enhance its suggestions. 
Currently, IBM Watson Health provides commercial software of the Watson program for genes, oncology, developing medications and managing healthcare [20]. IBM has cooperated with various private and scholarly foundations to use Watson in managing and studying patient care. For instance, IBM collaborated with WellPoint Corporation, in 2013, to train Watson in utilization management and collaborated with Memorial Sloan Kettering Cancer Center to train Watson in retrieving and rendering data associated with pulmonary carcinoma [21]. Furthermore, IBM and the large Indian Manipal Hospitals, in 2015, declared introducing IBM Watson for Oncology to screen data and offering clues for clinicians and patients to assist in finding individualized, evidencebased cancer managing alternatives. Additionally, this service, presented as clinician-induced professional second advice, is readily accessible by patients via Manipal Hospitals' web page [22]. Watson's capability for research and patient care, like many intelligent CDSS, necessitates physicians counting for the legal and ethical consequences of their adoption.

IBM produced Watson to facilitate and improve making decisions by clinicians via providing more certainty in diagnosis and management options for their patients [23]. Therefore, the program should not be judged as a definitive platform for making decisions that would substitute clinicians' opinion. The Food and Drug Administration (FDA) controls medications and devices' effectiveness and safety in the United States. Since Watson is not a device but a managing program regulated by healthcare professionals, currently it does not need restrictive inspection [24]. Nevertheless, supervision requisites might vary as Watson and other growing AI programs are adopted to make diagnostic or managing decisions with slight or no monitoring by clinicians [25]. Although Watson would support the precision of clinical decisions and enhance health results, adopting such a program might raise institutions and clinicians' responsibility. Technical advances produce chances for additional obvious mistakes, in diagnosis and management that may lead to ultimately harmful results compared to not adopting such techniques. For instance, Watson might suggest a certain therapeutic scheme which a clinician adopts meantime neglecting other patient data since believing that Watson, like other CDSS, had assessed these data. Hence, potential malpractice claims against caregivers [26].

Although technical approaches like Watson could enhance patients' results, they also can shortly add to an increased legal care standard, which can lead to accusing caregivers with carelessness [21]. While the care standard anticipations' could change, the technology effect on health results is yet not sufficiently recognized. For instance, if Watson could boost leukemia diagnostic precision and therapeutic suggestions', hence anticipations that caregivers who ask Watson would obtain the correct diagnosis and therapeutic options would be elevated greatly. Nevertheless, a lack of clinical trials assessing each potential diagnosis and managing option could restrict Watson's validity and utility. For example, the suggestions offered by the application may not be verified by adequate research to establish trust in clinicians who might be seen accountable for their diagnoses or managing decisions that could be found false or probably risky. Therefore, Watson users' shall balance between a perception of possible danger from increased trust in the application for each patient and a recognition that the application could also enhance their decisions.

Hence, prior to asking Watson, clinicians should realize the technological limitations of the application accessing and examining the required quality data to infer suggestions. Special issues with patient medical record could result in lacking or insufficient data. If Watson encounters faulty data, eventually it may lead to faulty or contradictory diagnosis and managing options. Still, adopting applications like Watson, leads to detect flaws, like mistakes in man input, which a human could normally miss. Evidently, a key advantage of applications like Watson is automatically finding patterns which not yet known to professionals, minimizing doubt and enhancing patient results [26]. Consequently, Watson could provide a conflicting suggestion compared to the preexisting medical criteria or it might oppose a clinician's perspective about the right decision. For instance, a medical criterion could be usually to order a certain therapy with a certain diagnosis, although a smart program like Watson might suggest another option (a non-standard therapy or no therapy). Correspondingly, clinicians should be capable of affirming their decision to comply or not with the provided option and to realize the possible medical and legal outcomes. Nonetheless, programs like Watson could and should be configured to utilize a ground rule, which may restrict suggestions to the preexisting medical criteria, thereby guaranteeing coherent suggestions with managing standards and already approved procedures.

Incompatibility is related also to the black-box dilemma, where both programmers and consumers could not recognize the program mechanism of action and hence the subsequent recommendations [24]. For instance, the machine-learning algorithm of Watson, without any clear clues, may produce incompatible recommendations to a clinician's opinion concerning diagnosis or prognosis. Consequently, it is vital ethically for the AI applications' programmers and 
consumers to realize or even clarify the algorithms' operating fundamental rules to minimize harming patients. A prerequisite audit trail with the least possible details to outline the decision procedure may be a method of solving the black-box dilemma and securing the confidence of the community [20]. Furthermore, systems like Watson could bring unreasonable patient predictions about results. For instance, a patient could be too ambitious regarding novel therapy since the clinician asked Watson, a supposedly very brilliant computer, which could perform superiorly to man. For the confidence-based patient-provider relationship, patients shall be briefed, by the treating clinicians, concerning the techniques utilized for decision-making regarding their health. Hence, medical computers, with their restrictions, should be recognized as a means for aiding but not making decisions.

Traditionally, employing technical clinical decision support applications is challenging [27, 28]. Some constraints are caused by data technical restrictions, implementing problems such as process integration and utility, and clinicians' perceiving technology while promised periods and functionality have not been attained [28]. For instance, IBM Watson Health has been blamed for not satisfying hopes of the programs' capability to reshape cancer therapy and results [29, 30]. Obviously, smart computerized applications like Watson would be significantly demanded since complicated clinical data and rapid growth. For instance, IBM has indicated that in a lifespan, an individual would yield 1 million gigabytes of medical data- comparable to larger than 300 million books [31]. Provided that the quantity of complicated clinical data, clinicians would rely on smart applications like Watson. Subsequently, it might be significantly regarded immoral, and hence resulting in accountability, for not seeking a second opinion from Watson or similarly smart applications, implying an established effectiveness in their intended purpose.

\section{FUTURE PERSPECTIVES}

Regardless drawbacks, early AI methods and technologies yet can afford thorough personal medical data and forecast community health hazards, and their medicinal or population health application is presumed to spike dramatically in the horizon. Accordingly, being a dynamic innovation field, precision medicine techniques can revolutionize:

- Preventive medicine by enabling each nonliving item such as slot machines to distinguish genomic codes, hence, they now ought to recommend with or against a candy bar for cutting diabetes later on [32].
- Robotic surgery in which robots may superbly operate on surgeries upon individuals. For instance, Da Vinci ${ }^{\circledR}$ lets surgeons work with augmented sight, agility, accuracy, and command. Additionally, robots might operate remotely on astronauts who are blocked in long-lived outer space operations [33]. Furthermore, inoculated microchips might operate on surgeries rather cutting into the human body.

AI shall pull essential data out of a patient's digital footprint. Initially, it shall enhance productivity and preserve time; however, after sufficient trials it shall likewise instantly direct managing patients. For instance, in a type 2 diabetic patient visit; now a physician devotes considerable duration examining biological samples, reviewing ambulatory files, and searching medical recommendations from several scattered applications. Nonetheless, AI might systematically formulate the highly crucial threats and measures with the help of the medical file of a patient. It might as well systematically turn the visit's recorded discussions into a summary report for the physician to accept or override. These systems might preserve much time and might be adopted too soon since they help physicians and not substituting them [34]. When these applications are properly verified, they might hold added accountability. For example, with type 2 diabetic patients, AI might set the personal onset level of statins, instead of a strictly outlined algorithm of a single size suits everyone, provided the inconveniences in the history of a patient. The studies needed for this individualized medicine might just be feasible via AI rationally analyzing big portions of clinical data. Additionally, since AI can, at the same time, examine millions of feeds; it might bear a profound function in the area of preventing diseases. AI might dynamically propose recommendations whenever it finds about a patient's chance of acquiring any complications related to diabetes permits action. Conversely, in the healthcare practice, it shall be unrealistic to burden individual personnel with the role of intensively tracking each diabetic patient's dates and test results in a timely manner.

AI-driven applications might as well convey professional assessment experience into primary care. For example, if a picture of a dermatological injury is enough to detect its causes competently, pictures might be taken at a general practitioner clinic and headed to an expert dermatological AI application for immediate assessment. Patients known to be of a reduced vulnerability could get rapid consolation whereas increasingly vulnerable patients could undergo less consultation waiting periods since health facilities could just be approached by chosen conditions. This 
model is not restricted to dermatological injuries as AI was promising in translating a number of various sorts of picture data involving ultrasound, optic scans and radiographs [35-37]. Some of these pictures might be caught with commonly accessible and fairly affordable devices.

Eventually, embedding AI techniques in the healthcare practice entails augmenting a reciprocally positive interaction among practitioners and $\mathrm{AI}$, because practitioners provide AI the vital required medical aspect to determine handling complicated medical conditions and AI provides practitioners better productivity and affordability. Across this procedure, it might be indispensable to ascertain that AI does not blur the human clinical essence since the greatest obstacle to AI's distributed implementation would be the common uncertainty to adapt potentially argumentative techniques [38].

\section{RESULTS}

Precision medicine entails adopting abundant disruptive technologies in medical practice, medications advancement, and healthcare delivery. AI can be employed in different varieties of clinical structured and unstructured data. Health intelligence applies tools and techniques from data science and AI to contribute helpful insights, decrease waiting time and loss, and boost service effectiveness, agility, efficiency, and productivity in medicine and health. Since the algorithms' predictive capabilities grow, machine learning shall display a vital factor in caregivers' work and patient care. While machine learning is becoming frequently prevalent in medical practice, attention has been raised respecting inclination in these systems' data, algorithms, and recommendations. Simply as medical care gets better for some; it may not get better for others. Employing AI suggests intricate legal issues concerning technology companies' and medical practitioners' accountability, especially if they could not justify recommendations produced by AI technology.

Eventually, the emerging novel approaches confronts health caregivers with common and occasionally brand-new ethical and legal implications. Several limitations about introducing and using programs like Watson hold resolving alternatives. With the availability of novel approaches, they obviously need time to investigate their medical usefulness and security. The inadequate literature on responsibility for innovation renders possibilities to think potential assumptions of AI for medical malpractice and products liability and innovative legal solutions for approaching accountability issues.

\section{CONCLUSIONS}

In order to warrant that the gains are maximized and the dangers are minimized, healthcare professionals should participate actively in developing this technology. It does not mean that healthcare providers shall abandon medicine and work with computational science. However, their clinical and medical knowledge are indispensable for engaging in AI development, formulating and adhering to standards and finding out limitations. The healthcare workers should be trained on AI utility and its necessity for efficiency. Medical institutions should prepare and educate their personnel on both the competencies and constraints of technical means meantime guaranteeing that patients are sufficiently aware of the methods such applications work to suggest recommendations regarding their care. It is improbable that smart applications like Watson would one day replace practitioners; however, they would greatly enhance health research and patient care. However, AI transforms healthcare delivery, practitioners will still treat patients and there will ever be a human component in the medical practice.

\section{REFERENCES}

[1] Firm hopes Big Data can personalize health care - The Boston Globe: 2013. https://www.bostonglobe.com/business/2013/05/12/pe rsonalized-medicine-goal-big-datascientist/28gTkXjCDj6Zh6KP5tpNBO/story.html. Accessed: 2019-06-07.

[2] Personalized Medicine and Psychiatry: Dream or Reality? 2013. https://www.psychiatrictimes.com/personalizedmedicine-and-psychiatry-dream-or-reality. Accessed: 2019-06-07.

[3] Douali, N. and Jaulent, M. 2012. Genomic and personalized medicine decision support system. 2012 IEEE International Conference on Complex Systems (ICCS) (Nov. 2012), 1-4.

[4] Collins, F.S. and Varmus, H. 2015. A new initiative on precision medicine. The New England Journal of Medicine. 372, 9 (Feb. 2015), 793-795. DOI= https://doi.org/10.1056/NEJMp1500523.

[5] Program Overview - All of Us | National Institutes of Health: https://allofus.nih.gov/about/about-all-usresearch-program. Accessed: 2019-06-06.

[6] Wilk, S. et al. 2017. A Data- and Expert-driven Decision Support Framework for Helping Patients Adhere to Therapy: Psychobehavioral Targets and Associated Interventions. Proceedings of the International Joint Workshop on Knowledge Representation for Health Care, Process-Oriented Information Systems in Health Care, Extraction and Processing of Rich Semantics from Medical Texts. MET Project. 53-66.

[7] Wilk, S. et al. 2017. Comprehensive mitigation 
framework for concurrent application of multiple clinical practice guidelines. Journal of Biomedical Informatics. 66, (Feb. 2017), 52-71. DOI= https://doi.org/10.1016/j.jbi.2016.12.002.

[8] Dimitriou, N. et al. 2018. A principled machine learning framework improves accuracy of stage II colorectal cancer prognosis. npj Digital Medicine. 1, 1 (Oct. 2018), 52. DOI= https://doi.org/10.1038/s41746018-0057-x.

[9] November 2018 | TOP500 Supercomputer Sites: $2018 . \quad$ https://www.top500.org/lists/2018/11/. Accessed: 2019-06-05.

[10] Esteva, A. et al. 2017. Dermatologist-level classification of skin cancer with deep neural networks. Nature. 542, 7639 (02 2017), 115-118. DOI= https://doi.org/10.1038/nature21056.

[11] Luo, G. et al. 2016. A novel left ventricular volumes prediction method based on deep learning network in cardiac MRI. 2016 Computing in Cardiology Conference (CinC) (Sep. 2016), 89-92.

[12] Wang, D. et al. 2016. Deep Learning for Identifying Metastatic Breast Cancer. arXiv:1606.05718 [cs, qbio]. (Jun. 2016).

[13] Bostrom, N. 2014. Superintelligence: Paths, Dangers, Strategies. Oxford University Press, Inc.

[14] Top Artificial Intelligence Companies in Healthcare to Keep an Eye On: 2017. https://medicalfuturist.com/top-artificial-intelligencecompanies-in-healthcare. Accessed: 2019-06-04.

[15] Goddard, K. et al. 2012. Automation bias: a systematic review of frequency, effect mediators, and mitigators. Journal of the American Medical Informatics Association: JAMIA. 19, 1 (Feb. 2012), 121-127. DOI= https://doi.org/10.1136/amiajnl-2011000089 .

[16] Anderson, M. and Anderson, S.L. 2019. How Should AI Be Developed, Validated, and Implemented in Patient Care? AMA Journal of Ethics. 21, 2 (Feb. 2019), $\quad 125-130 . \quad$ DOI= https://doi.org/10.1001/amajethics.2019.125.

[17] Lazer, D. et al. 2014. The Parable of Google Flu: Traps in Big Data Analysis. Science. 343, 6176 (Mar. 2014), 1203-1205. DOI= https://doi.org/10.1126/science.1248506.

[18] Luxton, D.D. 2016. Chapter 1 - An Introduction to Artificial Intelligence in Behavioral and Mental Health Care. Artificial Intelligence in Behavioral and Mental Health Care. D.D. Luxton, ed. Academic Press. 1-26.

[19] Ferrucci, D. et al. 2010. Building Watson: An Overview of the DeepQA Project. AI Magazine. 31, 3 (Jul. 2010), 59-79. DOI= https://doi.org/10.1609/aimag.v31i3.2303.

[20] Empowering Heroes, Transforming Health: 2014. http://www.merge.com. Accessed: 2019-06-30.

[21] IBM Watson Hard At Work: New Breakthroughs Transform Quality Care for Patients: 2013. https://www.mskcc.org/press-releases/ibm-watsonhard-work-new-breakthroughs-transform-qualitycare-patients. Accessed: 2019-06-30.

[22] Somashekhar, S.P. et al. 2018. Watson for Oncology and breast cancer treatment recommendations: agreement with an expert multidisciplinary tumor board. Annals of Oncology: Official Journal of the European Society for Medical Oncology. 29, 2 (01 2018), 418-423. DOI= https://doi.org/10.1093/annonc/mdx 781 .

[23] Otake, T. 2016. IBM big data used for rapid diagnosis of rare leukemia case in Japan. The Japan Times Online.

[24] Health, C. for D. and R. 2019. FDASIA Health IT Report. FDA. (Feb. 2019).

[25] Luxton, D.D. et al. 2016. Chapter 11 - Ethical Issues and Artificial Intelligence Technologies in Behavioral and Mental Health Care. Artificial Intelligence in Behavioral and Mental Health Care. D.D. Luxton, ed. Academic Press. 255-276.

[26] Luxton, D.D. 2019. Should Watson Be Consulted for a Second Opinion? AMA Journal of Ethics. 21, 2 (Feb. 2019), 131-137. DOI= https://doi.org/10.1001/amajethics.2019.131.

[27] Garg, A.X. et al. 2005. Effects of computerized clinical decision support systems on practitioner performance and patient outcomes: a systematic review. JAMA. 293, 10 (Mar. 2005), 1223-1238. DOI= $\underline{\text { https://doi.org/10.1001/jama.293.10.1223. }}$.

[28] McCullagh, L.J. et al. 2014. User centered clinical decision support tools: adoption across clinician training level. Applied Clinical Informatics. 5, 4 (2014), 1015-1025. DOI= https://doi.org/10.4338/ACI-2014-05-RA-0048.

[29] Ross, C. 2017. IBM pitched Watson as a revolution in cancer care. It's nowhere close. STAT.

[30] What will it take for IBM's Watson technology to stop being a dud in health care? 2017. https://www.technologyreview.com/s/607965/areality-check-for-ibms-ai-ambitions/. Accessed: 201907-10.

[31] IBM and Partners to Transform Personal Health with Watson and Open Cloud: 2015. https://www.prnewswire.com/news-releases/ibm-andpartners-to-transform-personal-health-with-watsonand-open-cloud-300065025.html. Accessed: 2019-0710.

[32] Awwalu, J. et al. 2015. Artificial Intelligence in Personalized Medicine Application of AI Algorithms in Solving Personalized Medicine Problems. International Journal of Computer Theory and Engineering. 7, 6 (Dec. 2015), 439-443. DOI= https://doi.org/10.7763/IJCTE.2015.V7.999.

[33] Small Robot Surgeon Designed to Work Inside Astronauts' Bodies: 2014. https://spectrum.ieee.org/tech-talk/robotics/medicalrobots/tiny-robot-surgeon-designed-to-work-inside- 
astronauts-bodies. Accessed: 2019-06-07.

[34] Buch, V.H. et al. 2018. Artificial intelligence in medicine: current trends and future possibilities. The British Journal of General Practice. 68, 668 (Mar. 2018), 143-144.

$\mathrm{DOI}=$ https://doi.org/10.3399/bigp18X695213.

[35] Alphabet's DeepMind uses A.I. to detect signs of eye disease: 2018. https://www.cnbc.com/2018/08/14/deepmind-uses-aito-detect-signs-of-eye-disease.html. Accessed: 201906-17.

[36] Chen, H. et al. 2017. Ultrasound Standard Plane
Detection Using a Composite Neural Network Framework. IEEE Transactions on Cybernetics. 47, 6 (Jun. 2017), 1576-1586. DOI= https://doi.org/10.1109/TCYB.2017.2685080.

[37] Lakhani, P. and Sundaram, B. 2017. Deep Learning at Chest Radiography: Automated Classification of Pulmonary Tuberculosis by Using Convolutional Neural Networks. Radiology. 284, 2 (2017), 574-582. DOI= https://doi.org/10.1148/radiol.2017162326.

[38] Naughton, J. 2017. Giving Google our private NHS data is simply illegal | John Naughton. The Guardian. 